\title{
The role of the Ti surface roughness in the self-ordering of TiO2 nanotubes: a detailed study of the growth mechanism
}

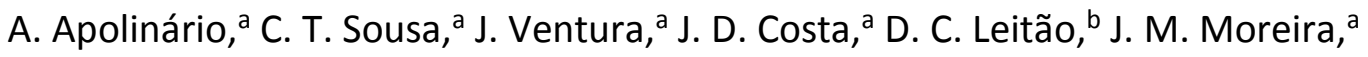 \\ J. B. Sousa, ${ }^{a}$ L. Andrade, ${ }^{c}$ A. M. Mendes ${ }^{c}$ and J. P. Araújo*a
}

aFIMUP and IN-Institute of Nanoscience and Nanotechnology, Departamento de Física e Astronomia, Faculdade de Ciências da Universidade do Porto, Rua do Campo Alegre, 678, 4169-007 Porto, Portugal. E-mail: jearaujo@fc.up.pt; jpearaujoc@fc.up.pt

bINESC-MN and IN, Rua Alves Redol 9, 1000-029 Lisboa, Portugal

'LEPAE, Dep. Engenharia Química-Faculdade de Engenharia, R. Dr Roberto Frias, 4200465 Porto, Portugal

Highly ordered TiO2 nanotubes (NTs) were synthesized by electrochemical anodization of Ti foils. We investigated the effect of the Ti surface roughness (applying different pretreatments prior to the anodization) on the length, growth rate and degree of selforganization of the obtained NT arrays. The mechanisms related to the TiO2 NT formation and growth were correlated not only with the corresponding anodization curves but also with their appropriate derivatives ( $1^{\text {st }}$ order) and suitable integrated and/or obtained parameters, to reveal the onset and end of different electrochemical regimes. This enables an in-depth interpretation (and physical-chemical insight), for different levels of surface roughness and topographic features. We found that pretreatments lead to an extremely small Ti surface roughness, offer an enhanced NT length and also provide a significant improvement in the template organization quality (highly ordered hexagonal NT arrays over larger areas), due to the optimized surface topography. We present a new statistical approach for evaluating highly ordered hexagonal NT array areas. Large domains with ideally arranged nanotube structures represented by a hexagonal closely packed array were obtained $\left(6.61 \mu \mathrm{m}^{2}\right)$, close to the smallest grain diameter of the Ti foil and three times larger than those so far reported in the literature. The use of optimized pre-treatments then allowed avoiding a second anodization step, ultimately leading to highly hexagonal self-ordered samples with large organized domains at reduced time and cost. 


\section{Introduction}

Electrochemical anodization of Ti to obtain highly ordered nanotube (NT) arrays of anodic TiO2 has gained much importance due to its large number of applications, such as photo-electrochemical cells for $\mathrm{H} 2$ production (water splitting), ${ }^{1}$ supercapacitors, ${ }^{2}$ biosensors ${ }^{3}$ and particularly for dye-sensitized solar cells (DSCs). ${ }^{4,5}$ In the latter case, the 1D architecture of the TiO2 NT structure provides a direct and efficient pathway for electrons up to the energy surface where they are collected. ${ }^{1}$ TiO2 NTs can be easily obtained by electrochemical anodization of Ti in fluoride containing electrolytes. Since the first report on anodic TiO2 NT synthesis in dilute fluoride electrolytes by Zwilling et al. in 1999 (with a limited thickness of $500 \mathrm{~nm}$ ), great effort has been made to increase the NT length. ${ }^{6}$ Ethylene glycol-based electrolytes have become the main choice for the growth of long (hundreds of microns) and ordered arrays of TiO2 NTs. ${ }^{7-13}$ More recently, NTs with a length of $150 \mu \mathrm{m}$ were obtained in $1 \mathrm{~h}$ of anodization with ethylene glycol electrolytes containing lactic acid. ${ }^{14}$ However, differently from the case of Al anodization, several reports have indicated that the length of TiO2 NTs reaches a maximum after a given anodizing time. This is attributed to the onset of an additional chemical dissolution process at the NT top and to the progressive failure of the dissolution at the NT bottom. ${ }^{9,15-19}$ The TiO2 NT length dependence on the oxide/dissolution balances on the NT bottom as well as the chemical dissolution on their top is a process strongly influenced by other factors besides the electrolyte type and anodization time, namely the electrolyte concentration, $\mathrm{pH}$, anodization temperature, and applied potentials, which have also been studied..$^{7-15,20-22}$ Other studies analyzed the effect of $\mathrm{NH}_{4} \mathrm{~F}$ and $\mathrm{H}_{2} \mathrm{O}$ concentrations (spanning $0.1-0.5$ wt\% and 2-15\%, respectively) with ethylene glycol-based electrolytes on the NT length, $8,11,12,16$ with the fastest growth rate obtained for $2 \%$ of $\mathrm{H}_{2} \mathrm{O}$ and $0.3 \mathrm{wt} \%$ of $\mathrm{NH}_{4} \mathrm{~F}^{8}$ Furthermore, high anodization voltages lead to longer NTs, while for low voltages the chemical dissolution effects dominate the growth process and limit the NT growth. ${ }^{16,17,23}$ However, for highfield hard anodization processes (from 90 to $220 \mathrm{~V}$; giving $100 \mu \mathrm{m}$ NTs in only $4 \mathrm{~h}$ ) the NT diameters, wall and barrier layer thickness greatly increase as they grow in length. ${ }^{24}$ The diffusion path along the barrier layer oxide for the ions involved in the NT formation is then significantly extended. This leads to a non-steady-state equilibrium between oxide formation and dissolution rate and to the deceleration of the NT growth. ${ }^{24,25}$ In contrast to the factors that increase the NT length, few studies targeted the organization and final ordering of the TiO2 NTs in a closely packed hexagonal array. ${ }^{25-29}$ Structural order is fundamental for several applications, particularly for efficient DSCs with maximized charge transport and reduced recombination processes. ${ }^{30,31}$ This requires an order-degree comparable to that already achieved for anodic alumina oxide (AAO) nano-porous templates (10\% porosity rule). ${ }^{32}$ In TiO2 NTs the order is mainly influenced by the Ti foil purity, the anodization voltage ${ }^{26}$ and the local initial surface morphology of the Ti substrate. For an irregular surface with many inter-grains, the inhomogeneous distribution of the electric field (being higher in the valleys) provides preferential sites for NT growth. ${ }^{33}$ However, regular or periodic dimples in a valve metal can lead to an improvement of the organization degree of NT arrays, inhibiting their 
random nucleation. The two-step anodization process, which is crucial and mandatory to improve organization in $A A O,,^{34,35}$ was also used to improve the structural order of TiO2 NT arrays. ${ }^{25-27}$ On the other hand, the pre-treatment of the initial metal foil was shown to be a simple and efficient method to induce a high degree of organization in AAO templates. ${ }^{36}$ In the TiO2 NT array case, studies using pretreatments such as electropolishing (EP) or mechanical polishing (MP) indicated that a Ti smooth surface is crucial to obtain highly ordered NT arrays since it ensures a uniform electric field distribution over the Ti surface during the anodization. ${ }^{27-29,37}$ In particular, Lee et al. showed that a decrease of the surface roughness by EP leads to NTs with a more uniform length and a higher density, but almost no hexagonal NT domains were observed. ${ }^{27,28}$ So far high hexagonal ordering has only been achieved using expensive techniques like ion beam or nanoimprint lithography. ${ }^{38,39}$ Furthermore, a direct correlation with the underlying formation and growth mechanisms, using a high resolution analysis of the anodization curves, was over-looked in previous reports. ${ }^{8,25,27-29,37,38}$ In this work, we describe the microscopic mechanisms involved in the TiO2 NT nucleation, formation and growth, which are key-issues of the degree of self-organization and growth rate. This information was extracted from a detailed interpretation of the anodization curves: current density curves $\mathrm{j}(\mathrm{t})$, their derivative $(\mathrm{dj} / \mathrm{dt})$, barrier layer thickness $(\delta \mathrm{b})$ and charge (Q). This procedure was systematically applied to the study of NT arrays obtained from initial Ti foils with different surface roughnesses (employing different pre-treatments). We were then able to achieve an integrated view on the processes underlying the formation of the NTs and their direct impact on the length and organization of the obtained arrays. We focused on the factors limiting the NT length and the critical balance between oxidation/dissolution processes, establishing a relationship between these phenomena and identifiable parameters induced by the different pre-treatments employed. A new statistical methodology to characterize ordering by SEM images is presented. This work reveals a new overriding anodization parameter capable of inducing a higher growth rate and improved self-organization of TiO2 NT arrays: the Ti surface roughness prior to the anodization, a parameter which has so far been overlooked. By tuning the Ti roughness using adequate pre-treatments, a single-step anodization process readily produces highly ordered hexagonal closely packed arrays of TiO2 NTs with lengths above $100 \mu \mathrm{m}$.

\section{Experimental details}

Ti foils (0.127 mm thick, 99.99+\% purity from Alfa Aesar) were ultrasonically cleaned in ethanol and deionized water for 10 minutes. Prior to the anodization, three different pre-treatments were performed on $1 \mathrm{~cm} 2$ pieces of the as-rolled (AR) Ti foil: (i) chemical etching (CE) in a 4\% (wt\%) HF aqueous solution for $15 \mathrm{~min}$ at room temperature;24 (ii) mechanical polishing (MP) on a plane disc at $150 \mathrm{rpm}$ in three steps of $10 \mathrm{~min}$ each. In the first two steps we used an ultrapol-cloth with 6 and $3 \mathrm{~mm}$ diamond particle suspensions; in the third step a silica colloidal suspension $(0.05 \mu \mathrm{m})$ with $\mathrm{H}_{2} \mathrm{O}_{2}(30 \%$ concentration in volume) on a microcloth was used; (iii) electropolishing (EP) in a $\mathrm{H} 2 \mathrm{SO} 4-\mathrm{HF}(8: 3)$ solution, with an applied potential of $10 \mathrm{~V}$ between the Ti foil and an 
inert Pt mesh (distance $2.5 \mathrm{~cm}$ ) for $4 \mathrm{~min}$; the solution was mechanically stirred (300 rpm) and kept at room temperature. ${ }^{40}$

After each pre-treatment the corresponding Ti foil samples, as well as an AR Ti sample without treatment, were analyzed using a Nanoscope Multimode Atomic Force Microscope from Veeco Instruments to study the surface roughness (Rq). Subsequently, the samples were electrochemically anodized in an in-house made anodization cell (twoelectrodes), where Ti acted as the anode and an inert Pt mesh was used as the cathode. The time evolution of the current density $[\mathrm{j}(\mathrm{t})]$ was monitored during the anodization using a Keithley 2004 Sourcemeter remotely controlled by a Lab-View application (using a $100 \mathrm{~ms}$ acquisition step in the first $5 \mathrm{~min}$ ). Electrochemical anodization was carried out for each sample at a constant potential of $60 \mathrm{~V}$ for $17 \mathrm{~h}$, in a freshly prepared ethylene glycol solution containing $\mathrm{NH}_{4} \mathrm{~F}(0.3 \mathrm{wt} \%)$ and $\mathrm{H}_{2} \mathrm{O}(2 \mathrm{wt} \%)$ at room temperature with mechanical stirring.8 The NT morphology was evaluated by FEI Quanta 400FEG Field Emission Scanning Electron Microscopy (SEM) using the cross-sectional (for the NT length calculation) and surface top and bottom views. The bottom views were prepared by natural peeling-off, becoming a free-standing non-curling membrane (detachment occurs without any assistance or other methods as described elsewhere). ${ }^{31,41}$ From the SEM images, a statistical analysis was performed enabling a quantitative evaluation of the organization pattern and NT structure dimensions.

\section{Results and discussion}

\subsection{Ti surface topography analyses}

The surface topography of the Ti samples with different pre-treatments (and one asrolled) was investigated by Atomic Force Microscopy (AFM). Fig. 1 shows representative $1 \mu \mathrm{m} 2$ images of such Ti surfaces. The average surface roughness was extracted from the topography, defined as the root-mean-square value of the image pixel height. The AR sample presents a significant surface roughness of $8.3 \mathrm{~nm}$ [Fig. 1(a)]. Afer the CE pretreatment, the $\mathrm{Ti}$ foil showed a highly damaged surface, characteristic of a chemical attack, giving a higher etching rate on surface defects, namely grain boundaries. ${ }^{29}$ This led to the highest Rq value of all the studied samples [37nm ;Fig.1(b)].In contrast, a significantly smoother surface was obtained in the MP and EP samples with the Rq of $1.0 \mathrm{~nm}$ and $2.4 \mathrm{~nm}$, respectively [Fig. 1(c) and (d)]. With the MP pre-treatment, the Ti foil surface became smooth and fairly plane. On the other hand, the Ti surface after EP revealed a dimple-pattern structure. This shallow ripple-like structure is an EP characteristic of metals ${ }^{40}$ and can be used as a pre-pattern prior to anodization to obtain highly ordered oxide nanostructures, as shown in the case of Al. ${ }^{36,40,42-45}$ The electrochemical polishing of $\mathrm{Ti}$ is usually achieved in sulphuric acid-based electrolytes, which results in smooth surfaces. ${ }^{46}$ However, in this work the EP conditions used (electrolyte type and voltage) resulted in organized dimple patterns, ${ }^{40}$ with an interripple spacial period $\lambda=97 \mathrm{~nm}$ [Fig. $1(\mathrm{e})]$. 


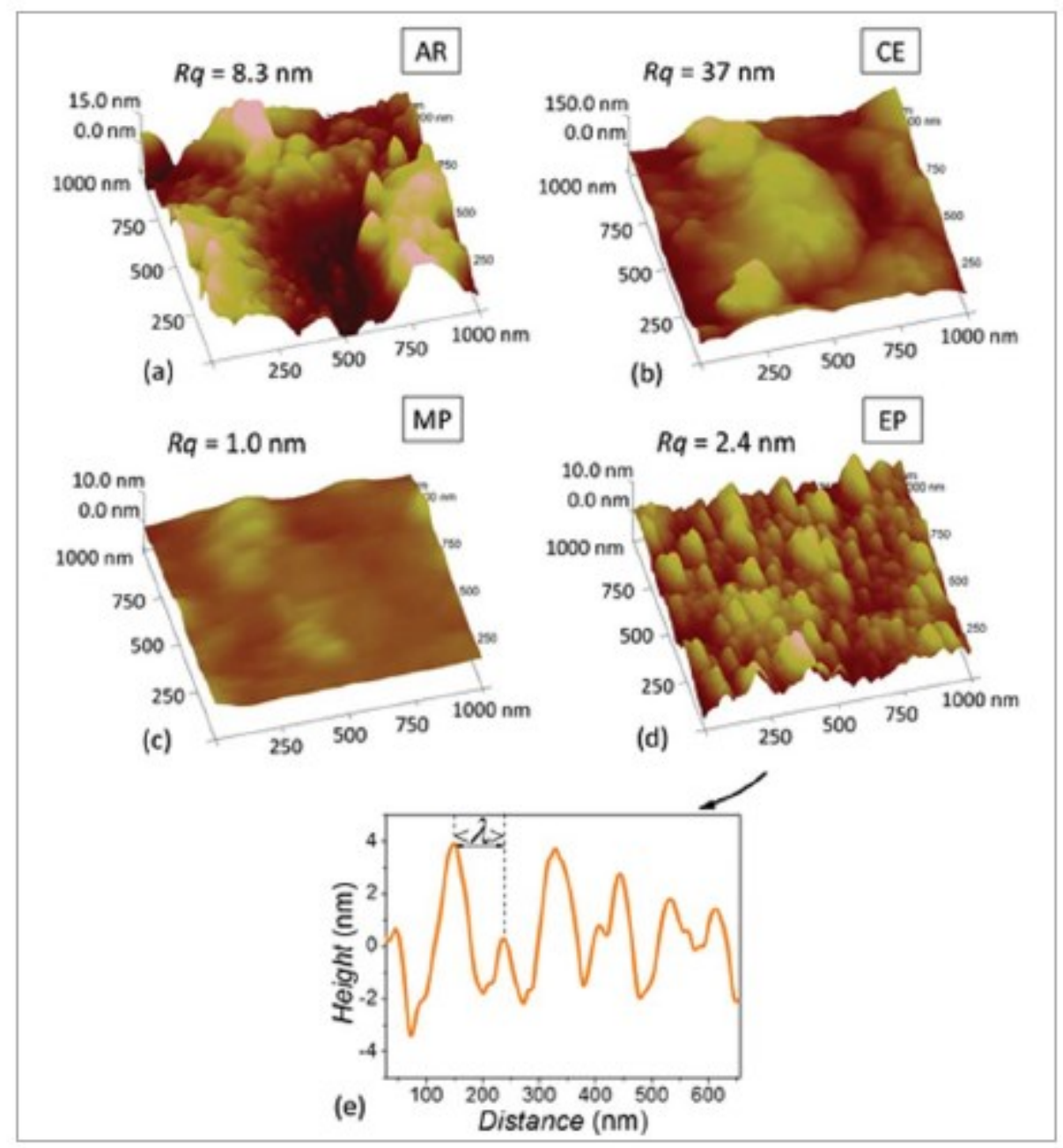

Fig. $1 \mathrm{Ti}$ surface topography prior to the anodization obtained using AFM and the corresponding Rq: (a) as-rolled, (b) chemical etching, (c) mechanical polishing and (d) electropolishing; (e) profile height for the EP sample.

\subsection{Growth mechanisms of anodization current density curves}

The mechanisms that lead to the formation and growth of the NTs can be monitored during the anodization process using the current density curves and the corresponding time derivative, as shown for the EP sample in Fig. 2(a) and (b). The evolution of the TiO2 barrier layer thickness $(\delta b)$ at the bottom of the NTs was also estimated from the $j(t)$ curve [Fig. 2(c)]. According to the high-field conduction theory, the current density (j) is related to the voltage $(\mathrm{V})$ drop across the barrier layer as follows:

$$
j=\alpha \mathrm{e}^{\beta \frac{V}{\delta_{\mathrm{b}}}},
$$


where $\alpha$ and $\beta$ are electrolyte and material dependent constants $\left(2.4 \times 10^{-37} \mathrm{Anm}^{-2}\right.$ and $56 \mathrm{~nm} \mathrm{~V}^{-1}$, at room temperature respectively) and the $(\mathrm{V} / \delta \mathrm{b})$ ratio is the effective electric-field across $\delta b .{ }^{16,25,47}$ From eqn (1) we obtain,

$$
\delta_{\mathrm{b}}=\frac{\beta V}{\ln \left(\frac{j}{\alpha}\right)},
$$

during the anodization. Note that even if the effective area changes during the anodization, its impact on the extracted value $\delta b$ is small, justifying the use of eqn (1). Since the electrical resistance $(\mathrm{R})$ across the Pt electrode-electrolyte-Ti electrode is initially small, the current density starts with a large value, $\mathrm{j}(\mathrm{t} \approx 0) \approx 65 \mathrm{~mA} \mathrm{~cm}^{-2}$ [Fig. 2(a)]. Then, a continuous TiO2 barrier layer is rapidly formed, substantially increasing $R$ (rapid j decrease; stage 1) until a minimum (jmin) is reached (stage 2). This minimum corresponds to the maximum of $\delta b$. The subsequent increase of $j$ (stage 3 ) indicates a $\delta b$ decrease, which persists until j reaches a maximum (jmax) and $\delta b$ reaches a local minimum (stage 4). From this point onwards new electrochemical processes come into play, as evidenced from the slow j decrease, indicating a slow-rate increase of $\delta b$ (stage 5).

Commonly, jmin is associated with the onset of NT nucleation, ${ }^{34}$ likely on the surface valley-type irregularities, where the higher electric field enhances oxide dissolution and hole formation. To deeply study the effects of topography on NT nucleation and growth, high resolution measurements of the time derivative (dj/dt vs. t) were used to investigate the subtle features present in $j(t)$ [Fig. 2(b)]. One sees that $d j / d t$ exhibits no singularity or change of behavior at $\mathrm{j}=\mathrm{jmin}$ and thus jmin cannot represent the beginning of the nucleation stage. In fact, considerably before reaching jmin [jl in Fig. $2(a)], d j / d t$ significantly slows down likely reflecting early random nucleation and pore formation by chemical dissolution ( $\mathrm{F}^{-}$ions) in favorable spots of the TiO2 surface. ${ }^{48}$ Although $\delta b$ is still increasing [Fig. 2(c)], indicating Ti oxidation dominance, the progressive emergence of the pores (the local dissolution of $\mathrm{db}$ gradually increases) reduces the effective barrier resistance-rate increase. There is then a competition between electrochemical Ti oxidation (increasing $\mathrm{R}$ ) at the Ti/oxide interface and $\mathrm{TiO}_{2}$ dissolution (lowering R) at the oxide/electrolyte interface. Accordingly jmin marks the balance between the two processes, thus the limiting oxide thickness ( $\delta$ bmax). In other words, oxidation is weakened by the increase of $\delta \mathrm{b}$, which progressively restrains $\mathrm{O}^{2-}$ electromigration through the thick $\mathrm{TiO} 2$ barrier, whereas the $\mathrm{F}^{-}$dissolution processes continue smoothly at the TiO2/electrolyte interface (Fig. 3). Consequently, $\delta b$ decreases after jmin (smaller rate of $\mathrm{Ti}$ oxidation), while the tube formation accelerates, as evidenced by the increase of $\mathrm{dj} / \mathrm{dt}$ up to the $\mathrm{j}(\mathrm{t}$ ) inflexion point at $\mathrm{jll}$ (maximum $\mathrm{dj} / \mathrm{dt}$ ). It is likely that a sufficient number of NTs already exist at jll, but the tube-formation process goes on until it effectively eliminates the available oxide regions for nucleation. Ultimately, saturation occurs at jmax, where $\mathrm{dj} / \mathrm{dt}=0$ (and $\delta \mathrm{b}$ is minimum). From this point onwards, the incipient NTs adjust and compete among themselves to improve the 
degree of order in a self-organized process. In this stage, $\delta b$ slightly increases, particularly between jmax and jlll, which marks the onset of a new regime. Notice that the nucleation time can now be defined as $\Delta t n=t n I I I-t n l$, where tnIII marks the kink at $\mathrm{jlll}$ and $\mathrm{tnl}$ marks the beginning of nucleation at jl. As the anodization proceeds, the formed NTs grow at a constant rate and $\delta b$ would remain constant if $\mathrm{Ti}$ oxidation balanced oxide dissolution. ${ }^{7,8,12,15}$ However, at this stage $(j<j$ lll), the oxidation/dissolution processes are not quite in equilibrium, the $\mathrm{TiO} 2$ dissolution being lower than its formation. This results in a smooth increase of $\delta b$ after the jlll kink and the consequent slow decay of $j(t)$ (stage 5). This is related to the lack of $F^{-}$ions at the NT bottoms, since they are difficult to replace by fresh ones due to the high viscosity of the electrolyte. As the NTs grow, large diffusion distances (lengths) must be travelled and thus longer times are required to renew the $\mathrm{F}^{-}$concentration at the pore bottom. Consequently, the oxidation at this stage becomes higher than the dissolution, leading to an increase of $\delta b$ that further inhibits the transport of $\mathrm{F}^{-}, \mathrm{Ti}^{4+}$ and $\mathrm{O}^{2-}$ ions across the barrier $^{12}$ (Fig. 3) and further limits NT growth (stage 6).

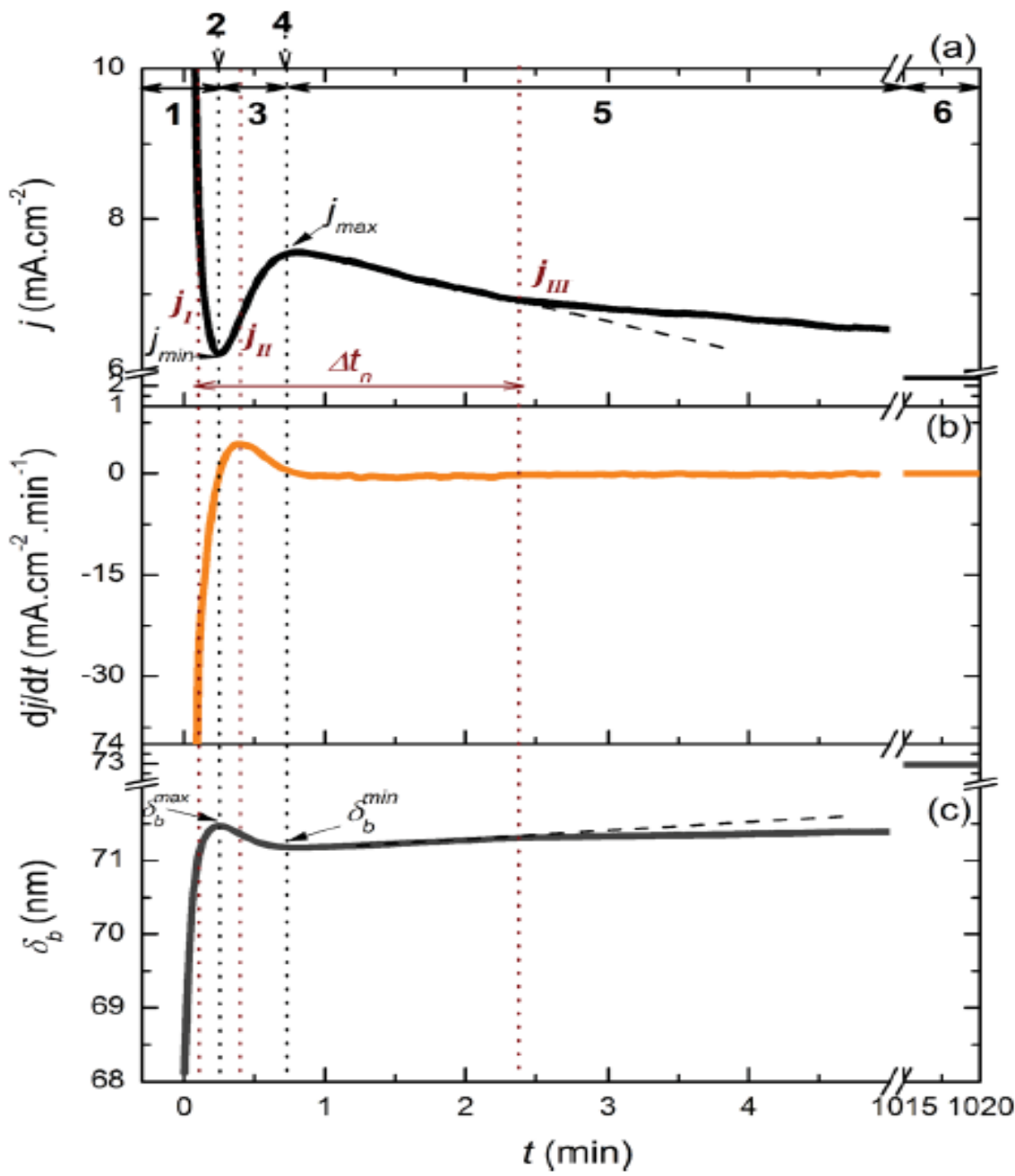

Fig. 2 (a) Transient period of the anodization curves for the EP sample; (b) the corresponding derivate and (c) $\delta b$ associated with different stages of the TiO2 NT growth mechanisms. 


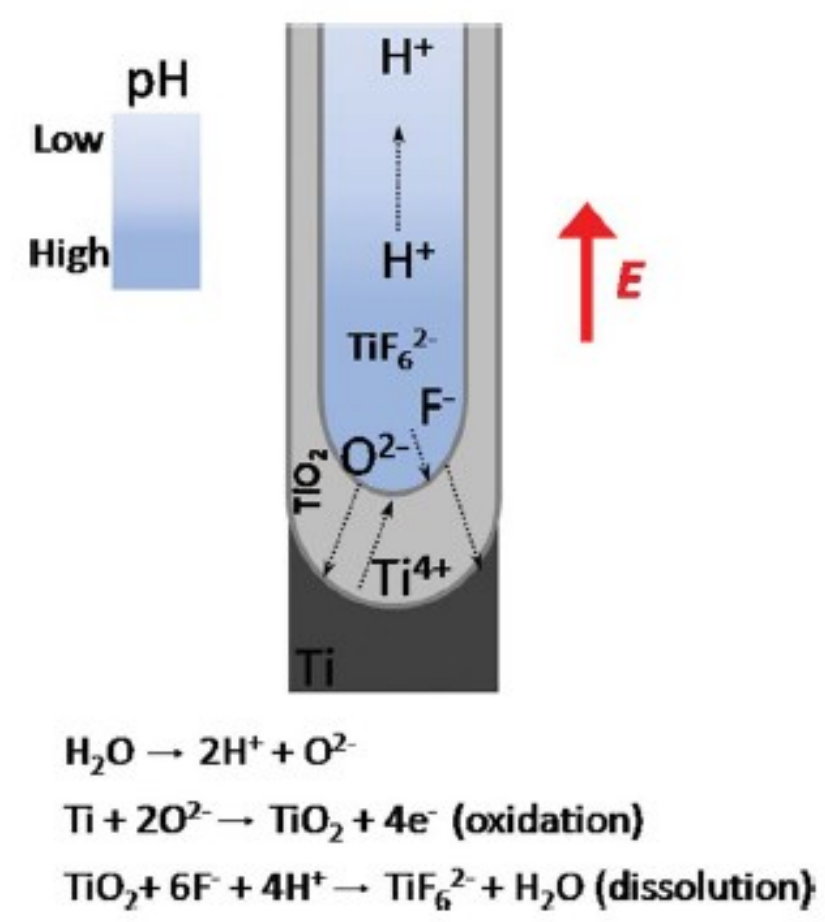

Fig. 3 Schematic diagram illustrating the ion diffusion profiles inside the NTs during the anodization. The dissolution of TIO2 occurs at both the top and the bottom of the NTs, while the oxidation occurs only at the bottom.

\section{Growth mechanisms for different levels of roughness.}

The $j(t)$ curves of the samples subjected to different pre-treatments used in this work are shown in Fig. 4(a). The $j(t)$ transient of the MP sample substantially differs from all others in two critical aspects: (i) an extended period of time over which low-rate pore nucleation smoothly takes place and (ii) the fairly small $j(t)$ values over such a region, indicating the growth of a thicker TiO2 barrier layer [Fig. 4(c)]. This is at first sight unexpected when compared to the EP sample, which presents similar Rq values ( $2.4 \mathrm{~nm}$ versus $1.0 \mathrm{~nm}$ of the MP sample). However, the MP sample has an almost flat and smooth surface, whereas the EP surface reveals unique periodical rippled structures [Fig. 1(d) and (e)]. These ripple structures act as nucleation points (valleys) so that nucleation occurs simultaneously on many sites, making the overall nucleation time fairly short. In opposition, the lack of topographic irregularities in the MP sample leads to longer nucleation times (longer $\Delta \mathrm{tn})$ and a fairly low jmin value $\left(\approx 1.5 \mathrm{~mA} \mathrm{~cm}^{-2}\right)$, i.e. a thicker $\delta b$. The rapid dissolution rate of the EP sample is clearly observed in the $\mathrm{dj} / \mathrm{dt}$ and $\delta b$ curves [Fig. $4(b)$ and (c)]: after jmin the pore dissolution accelerates as $\delta b$ decreases, with $\mathrm{dj} / \mathrm{dt}$ rapidly reaching its maximum value at the inflexion point ( $\mathrm{jll}$ ), appearing much earlier than for the MP sample. Additionally, the MP sample exhibits a much higher and broader $\delta$ bmax peak [Fig. 4(c)], confirming the much slower dissolution rate during the porous formation. The consequence is the absence of the typical shoulder-like behavior in $\mathrm{j}(\mathrm{t})$ ascribed to the competition between growing NTs and consequent organization quality, ${ }^{35,36}$ being more readily organized during the slow 
oxide-dissolution process. On the other hand, in the EP sample the vigorous faint dissolution rapidly leads to a pronounced jmax and a shoulder-like trend. Furthermore, in the inset of Fig. 4(c) we notice that at the end of the anodization, samples with lower $\mathrm{Rq}$ (MP and EP) exhibit a final $\delta b$-value lower than samples with higher Rq (CE and AR). Fig. 5 shows a SEM image (backscattered mode) of the MP sample, with $\delta b=73 \mathrm{~nm}$, in excellent accordance with the value obtained using the curve $\delta b(t)[F i g .4(c)]$ after $17 \mathrm{~h}$ of anodization. The $\mathrm{j}(\mathrm{t})$ curve of the AR sample presents a similar shape to that of the EP sample, i.e. a smaller $\Delta$ tn despite its larger Rq. This shows that both samples have enough easy-nucleation spots related to the topographic valleys. Nevertheless, the ripple EP valley structure favors earlier and faster organized nucleation (smaller $\Delta \mathrm{tn}$ ) than the broader random valleys of the AR sample. The previous analysis of the anodization curves of the MP, EP and AR samples clearly reveals the importance of the surface topography details (smooth, ripple-like and random broad valleys) in promoting effective nucleation spots and thus the earlier emergence of NT nucleation and lower $\Delta \operatorname{tn}: \Delta \operatorname{tn}(E P)<\Delta \operatorname{tn}(A R)<\Delta \operatorname{tn}(M P)$. Fig. 6 shows jmin and jmax - jmin as a function of $R q$ for the AR, EP and MP samples. With the decrease of Rq, jmin also decreases and higher jmax - jmin values are reached. Therefore, Ti surfaces with lower Rq lead to higher TiO2 oxide barriers ( $\delta$ bmax) and quality, since less defects are present in the primordial $\mathrm{Ti}$ surface, and the dominant oxidation process [at stage (1)] delays dissolution. Moreover, lower Rq leads to thinner $\delta$ bmin and $\delta$ b during the anodization, which allows higher $j(t)$ (due to a more uniform and optimized electric field and current) and consequently an increase of the NT growth rate.

The particular case of high roughness. The sample pre-treated with CE shows different $\mathrm{j}(\mathrm{t})$ behavior from those previously discussed (or shown in the literature) due to severe chemical etching effects (rise of Rq from $8.3 \mathrm{~nm}$ to $37 \mathrm{~nm}$; see Fig. 1). In particular, it is difficult to directly extract key-values such as $\Delta$ tn from the $j(t)$ curves. One simply observes a monotonic decrease of $j$ along the whole anodization period, indicative of stronger oxidation than dissolution processes. This explains the absence of the typical transient period with nucleation and dissolution stages, as well as jmax and jmin. The AFM topographic imaging was performed after $17 \mathrm{~h}$ of anodization and after removal of the TiO2 NT template (Fig. 7). The Ti surface revealed the characteristic pre-pattern left by the NTs, but different areas of organization and depth can be observed [zones 1 and 2 in Fig. 7(a)]. The regions with higher depth (zone 2) reached a higher anodization growth rate since these areas correspond to the primordial etched Ti surface with valleys (topographic minima). In contrast, the regions with hills had a lower anodization growth rate and showed a lower depth (zone 1). Therefore, the measured macroscopic $\mathrm{j}(\mathrm{t})$ curve has contributions from many different anodization zones. 


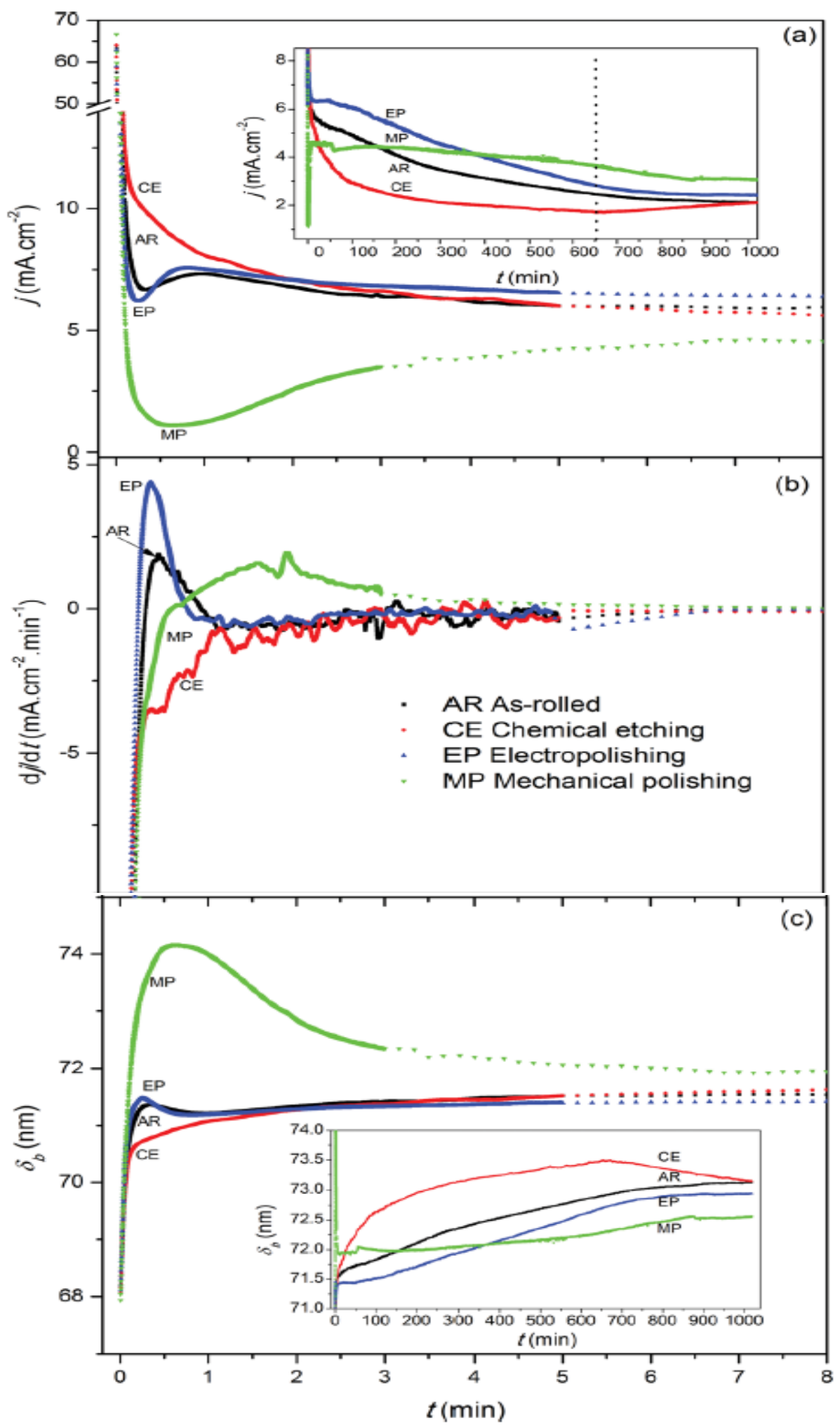

Fig. 4 (a) Current density anodization curve transient for $8 \mathrm{~min}$ of anodization (inset: 17 $h$ of anodization); (b) corresponding derivatives; (c) TIO2 barrier layer thickness ( $\delta b$ ) for 8 min of anodization (inset: $17 \mathrm{~h}$ of anodization) for the AR, CE, MP and EP samples. 


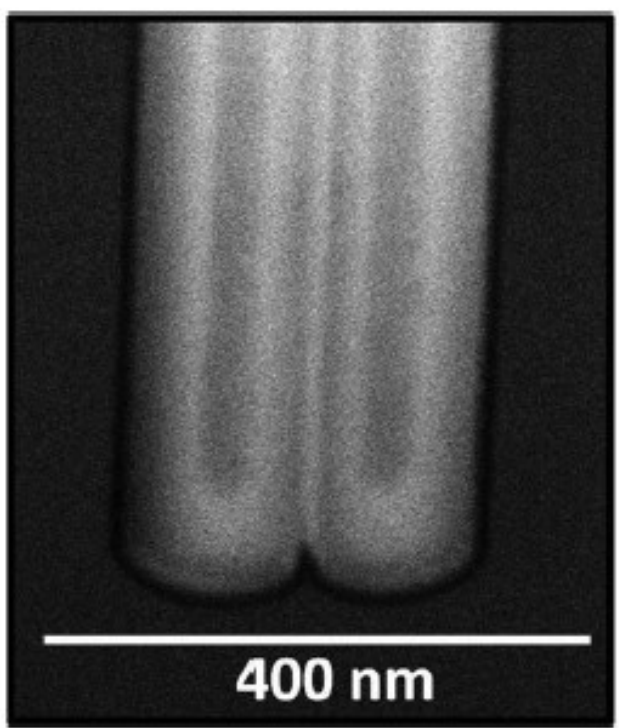

Fig. 5 Measurements of $\delta b$ after $17 \mathrm{~h}$ of anodization for the MP sample in SEM backscattered mode.

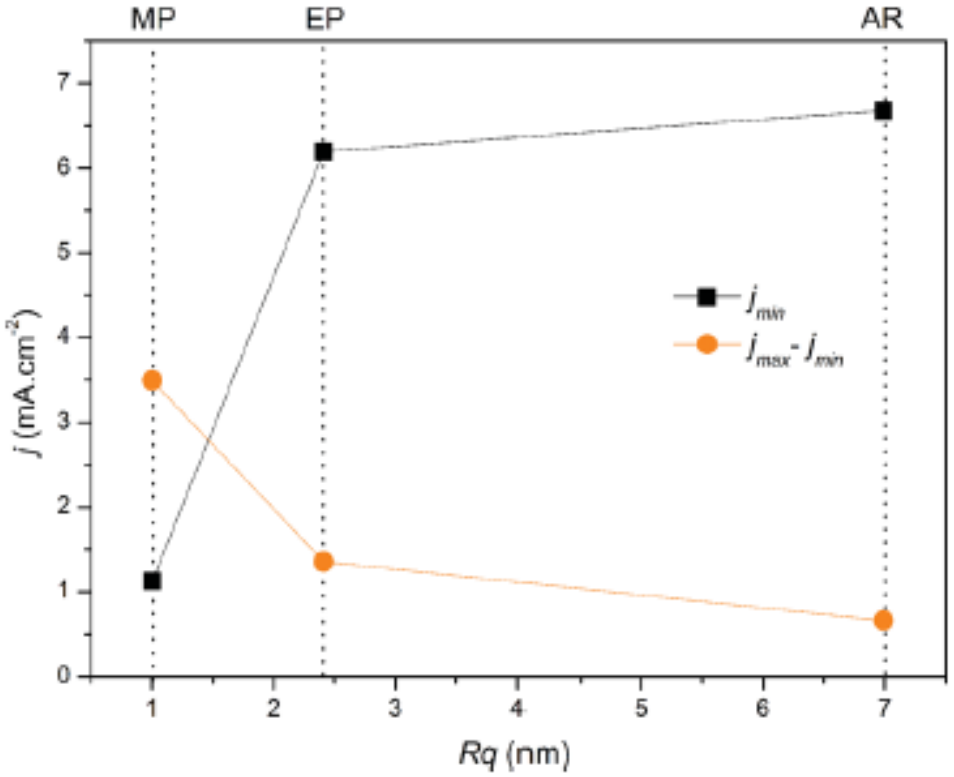

Fig. 6 jmin and jmax - jmin values as a function of the surface roughness for: AR, EP and MP samples. 


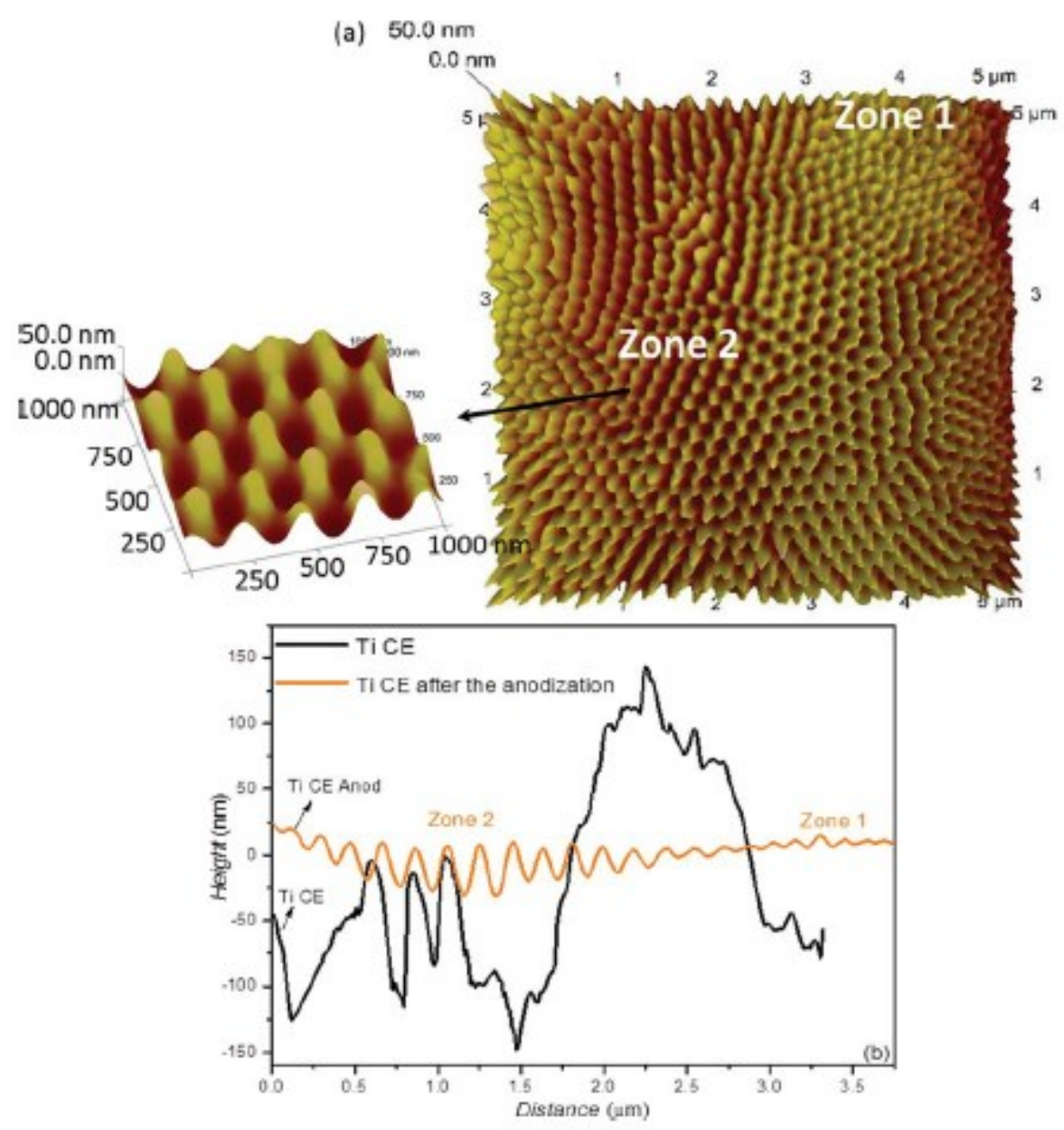

Fig. 7 (a) AFM image of the TI surface of the CE sample after $17 \mathrm{~h}$ of anodization and removal of the TIO2 NT template; after the anodization the Ti surface reveals the NT array hexagonal pattern. There are two different zones with different growth rates: zone 1 with a slower anodization growth rate and zone 2 with a higher anodization growth rate; (b) the corresponding section of the height profile.

\subsection{Growth rate of anodization charge curves}

Fig. 8 shows the charge curves $Q(t)$ obtained from the $j(t)$ data. The $Q(t)$ curves of all samples present non-linear shapes characteristic of non-steady state processes. The decrease of the slope during the $\mathrm{Ti}$ anodization is due to the lack of an oxidationdissolution balance (Ti oxidation is higher than $\mathrm{TiO} 2$ dissolution) that leads to an increase of $\delta b$ as the anodization proceeds [Fig. 4(c)].

The $Q(t)$ slope of the EP and MP samples is higher than that of AR and CE samples for the entire anodization period (Fig. 8). This behavior is related to the steady-state balance between the oxidation and dissolution processes in the EP and MP samples. Even though the MP and EP samples have almost the same final charge transfer of $3900 \mathrm{C}$ after $17 \mathrm{~h}$, the slope's trend is significantly different: whereas $Q(t)$ behavior in the MP sample is rather linear, providing an almost constant rate growth, the EP presents two distinct growth regimes. After 500 min of anodization time, the growth rate of the EP sample 
slows down due to the thicker $\delta$ b [Fig. 4(c)] ultimately being surpassed by the MP sample.

The charge transferred during the anodization process was found to be related to the surface $R q$. Lower $R q$ samples present higher $j(t)$ and $Q(t)$ values, since a more homogeneous electric field over the whole sample is reached, originating an oxidation/dissolution process more equally balanced and delaying the additional chemical effects that limit NT growth. As a higher final $Q(t)$ indicates higher NT template thickness (L), one expects $L(M P)>L(E P)>L(A R)>L(C E)$.

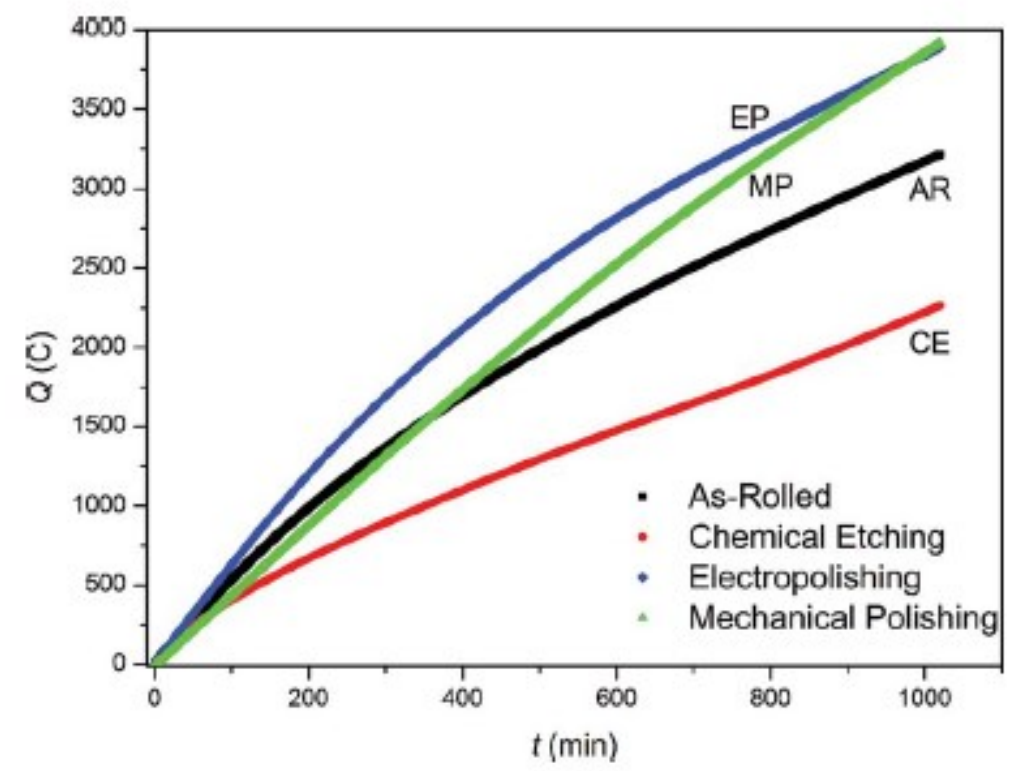

Fig. 8 Charge $[Q(t)]$ curves obtained by integrating the $j(t)$ anodization curves of the studied AR, CE, MP and EP samples; the EP sample slows down due to the thicker $\delta b$ [Fig. 4(c) inset] ultimately being surpassed by the MP sample.

\subsection{Nanotube growth}

Fig. 9 shows SEM cross-sectional and top view images of the NT templates for all samples after $17 \mathrm{~h}$ of anodization. The resulting template thicknesses are shown in Table 1 . The AR sample has a mean NT template thickness (L) of approximately $89 \mu \mathrm{m}$, while the CE sample has $(L)=83 \mu \mathrm{m}$. On the other hand, the EP and MP samples show a rapid NT growth and enhanced mean lengths of $125 \mu \mathrm{m}$ and $128 \mu \mathrm{m}$, respectively, i.e. a length increase of over $40 \%$ when compared with the AR and CE samples.

Comparing $(L)$ with the Rq results, one can infer that the surface topography is directly correlated with the NT growth and template thickness (Fig. 10). In fact, the pre-anodizing surface conditions are crucial to reach higher growth rates and thicker NTs, i.e. by decreasing Rq, longer NTs are obtained. It is important to notice that the large Rq value of the CE sample is responsible for the decrease of the NT growth rate and length up to $660 \mathrm{~min}$ anodization time. However, due to the acidity increase of the electrolyte at the NT top (top dissolution), the $\mathrm{F}^{-}$ions can then more easily reach the NT bottom, being readily available to again increase the NT growth rate. This is clearly seen in the $j(t)$ curve 
presented in the inset of Fig. 4(a), where a jincrease is observed at $t=660 \mathrm{~min}$, indicating that $L$ restarts to increase. The enhancement of the growth rate concerning EP and MP samples should result from the decrease of $\mathrm{Rq}$ and the presence of small nucleation sites (see Fig. 11). A smoother surface favors to the mobility of ions in the dominant anodization processes, promoting greater accessibility of $\mathrm{F}^{-}$ions that reach the Ti surface and thus favoring the dissolution processes. On the other hand, the additional chemical effects (namely $\mathrm{pH}$ decrease throughout the anodization) are retarded since the anodization growth limit (i.e. when oxidation surpasses the dissolution, causing a thick $\delta b)$ is delayed. Also, for samples with low $\mathrm{Rq}$, the $\delta \mathrm{b}$ increase during the anodization is slower [inset of Fig. 4(c)], which promotes ion electro-migration $\left(\mathrm{F}^{-}\right.$and $\left.\mathrm{O}^{2-}\right)$ through the $\delta b$, fundamental for the dissolution/oxidation balance processes. This is clearly visible in the MP and EP samples ( $\mathrm{Rq}$ of 1 and $2.4 \mathrm{~nm}$, respectively) that reach a template thickness enhancement of over $40 \%$, keeping an almost intact oxide nucleation layer after $17 \mathrm{~h}$ of anodization.

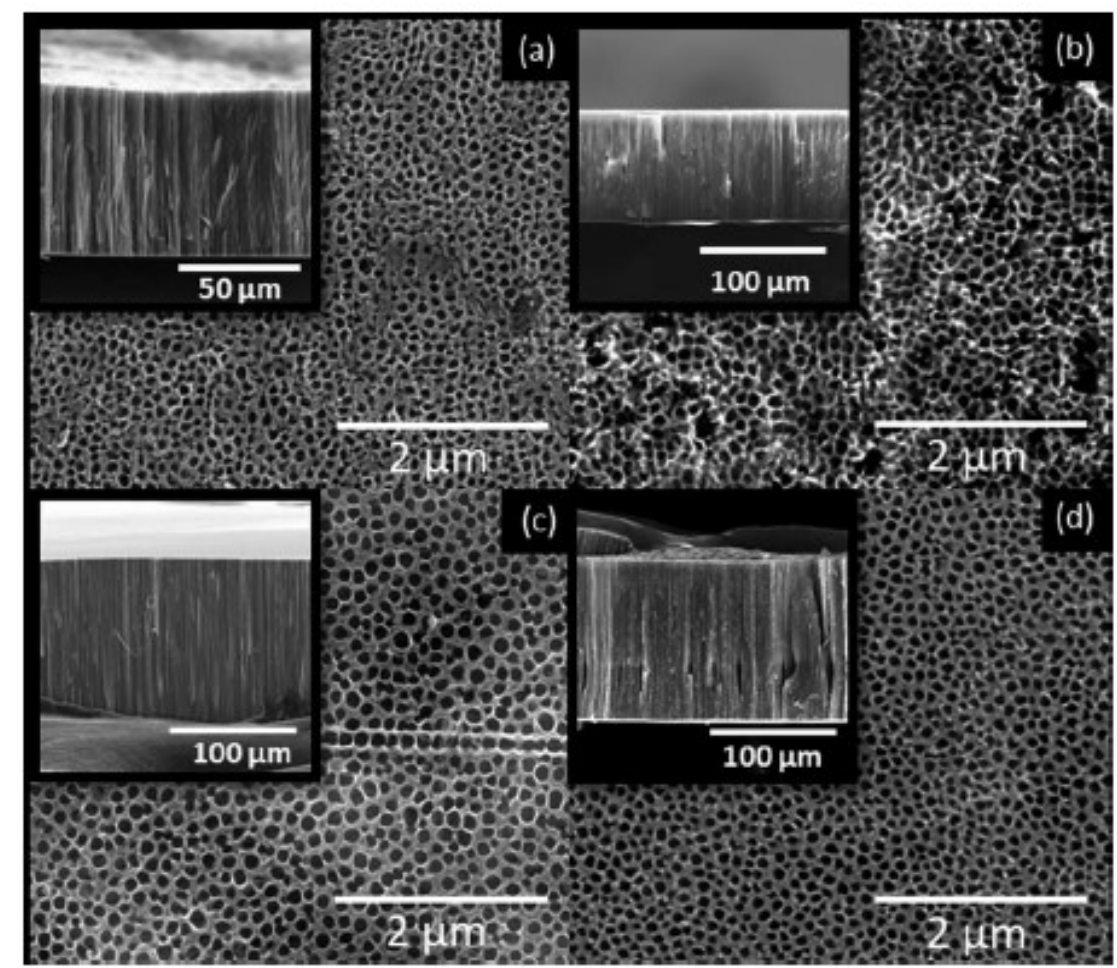

Fig. 9 Top view and cross-section (insets) of the TiO2 NT templates from the different samples after $17 \mathrm{~h}$ of anodization: (a) AR Ti foil, (b) CE, (c) MP and (d) EP. 


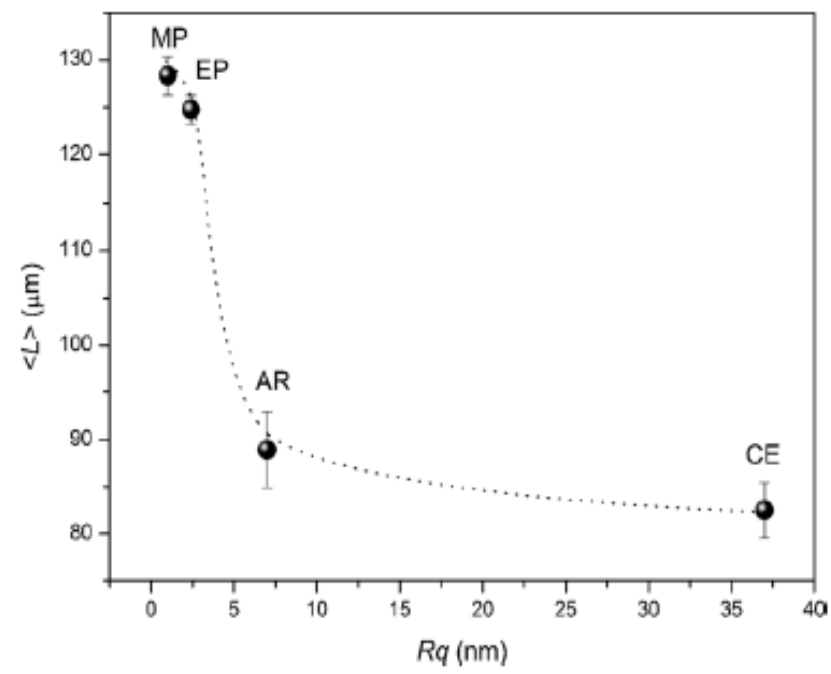

Fig. 10 TiO2 NT template thickness average (L) as a function of the Ti surface Rq.

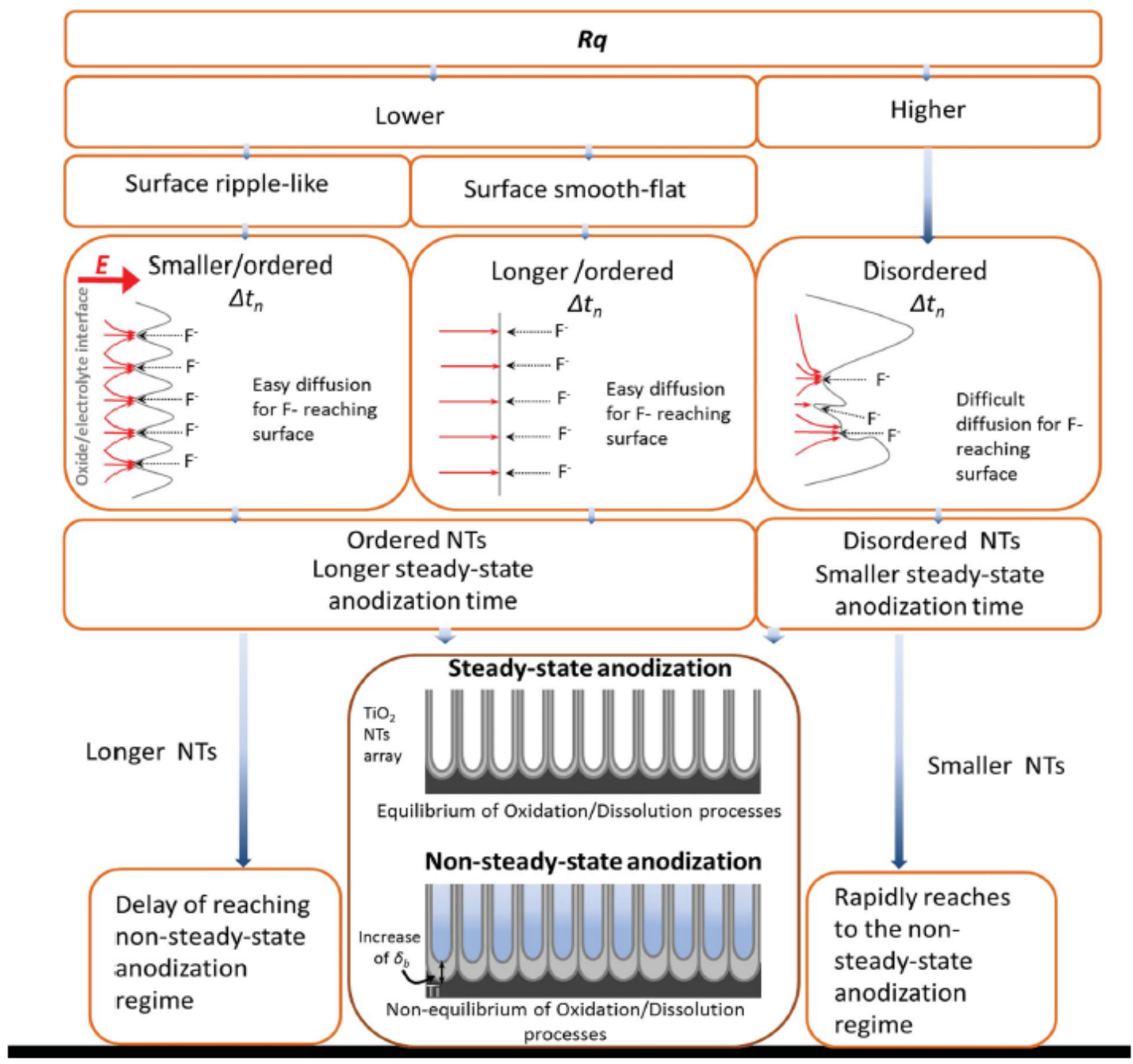

Fig. 11 Schematic diagram illustrating the influence of the Ti surface Rq (prior to the anodization) and topography on the growth mechanisms at the nucleation stage, NT ordering and length. To have longer and more ordered NTs steady-state anodization, that delays the $\delta b$ increase, is crucial. 


\subsection{Nanotube array organization}

To reveal the degree of organization of the produced NT arrays, we performed a statistical evaluation of SEM images (25 000x) of the NT template bottoms in a random area of each sample. Since the analyses of the top surface would show the initial random nucleation pores, the bottom analyses arise as a more adequate technique to evaluate the degree of organization. ${ }^{26,28}$

Based on this analysis the following parameters were quantified: (i) the NT hexagonal domain area (delimited area where the NTs have a hexagonal arrangement); (ii) the organized area (\% organized area/total evaluated area); and (iii) the NT density (number of NTs per unit area) and NT diameter distribution.

NT hexagonal domain area. The degree of order of the NT domains as a function of their hexagonal arrangement and individual shape was analyzed. ${ }^{26}$ The NT organization increases as the NTs tend to pack themselves in a closely packed hexagonal arrangement, leading to tubes with a hexagonal section. The deviations from this shape due to Ti defects and grain boundaries result in pentagons, heptagons or circular shapes. ${ }^{26}$ To quantify the order-degree of the domains, they were first classified into two types of classes: class 1 has a lower order-degree domain with an ideal Hexagonal Closely Packed Array (HCPA) structure, exhibiting NTs with diverse polygon shapes other than hexagonal; and class 2 domains having a higher order-degree domain with the same HCPA structure, but each NT exhibiting a hexagonal shape (Fig. 12). To calculate the domain areas, the HCPA areas of each SEM image were delimited and labeled using Image J. ${ }^{49}$ Fig. 13 shows SEM images of the bottoms of the NT layers of each sample (AR, CE, MP and EP). For each SEM image, besides the delimitation of these HCPA domains, the maximum sized ones were differently colored as class 1 (orange) and class 2 (green). Fig. 14 displays the statistical results of the analyzed SEM images. The CE sample displays large areas with completely disorganized NT arrays (darker areas) of small diameter [Fig. 13(b) and (f)]. Therefore, for the CE sample the statistical calculations were only possible on the small areas with NTs of large diameters, where some degree of organization occurs [inset of Fig. 13(b)]. These domains correspond to the valleys present in the $\mathrm{Ti}$ foils after the CE pre-treatment (see AFM images in Fig. 7) where the anodization rate is higher. In this sample, the obtained ordered array size presents an average value of 0.92 $\mu \mathrm{m} 2$. However, the organized area represents only $75 \%$ of the total area from the considered SEM image. The AR, MP and EP samples have the same organized area fraction ( $\approx 96 \%)$, with the MP and EP samples clearly having larger average domain sizes (0.80 and $0.92 \mu \mathrm{m} 2$, respectively) than the AR sample $(0.50 \mu \mathrm{m} 2)$. Note that, while the AR sample presents a class 1 maximum domain size of $1.8 \mu \mathrm{m} 2$, the EP and MP samples show much larger class 2 maximum domain sizes (6.61 and $4.32 \mu \mathrm{m} 2$, respectively). Therefore, a clear improvement of the NT quality and organization was obtained with the simple application of pre-treatments such as EP and MP in the Ti foil before anodization. The most relevant improvement found was the increase of the class 2 domain size from the EP sample. It is also important to mention that the area for the class 2 domains in the EP sample (or even in the MP sample) largely exceeds the maximum value of $1.83 \mu \mathrm{m} 2$ reported in the literature ${ }^{25}$ (or in the case of nano-porous template structures the reported domain area is about $0.25-1 \mu \mathrm{m} 2) .27 \mathrm{In}$ fact, the use 
of these pre-treatments pushed NT domain sizes close to the smallest grain diameter of commercial Ti sheets ( 5 to $50 \mu \mathrm{m}$ on average). ${ }^{50}$

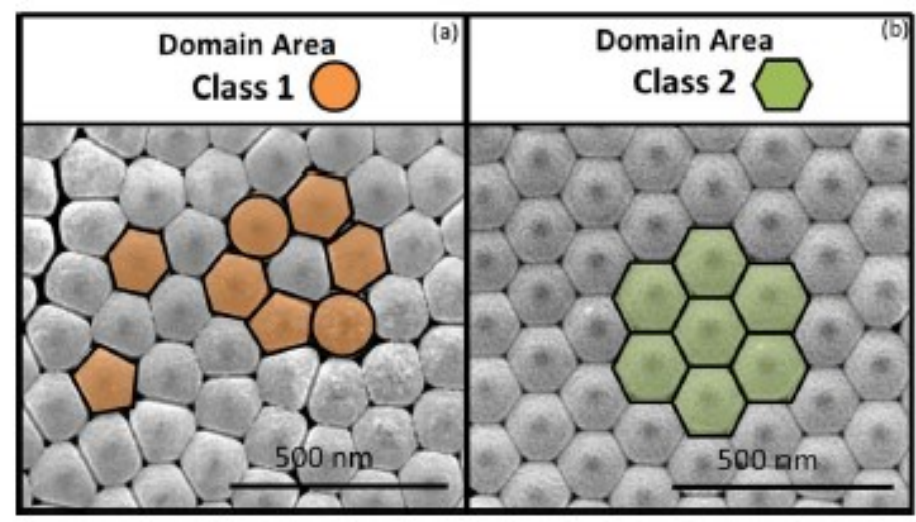

Fig. 12 Bottom TiO2 NTs for the two different classes. (a) Class 1: lower order (hexagonal arrangement; NTs without a hexagonal shape), (b) class 2: higher order (hexagonal arrangement; NTs with a hexagonal shape).

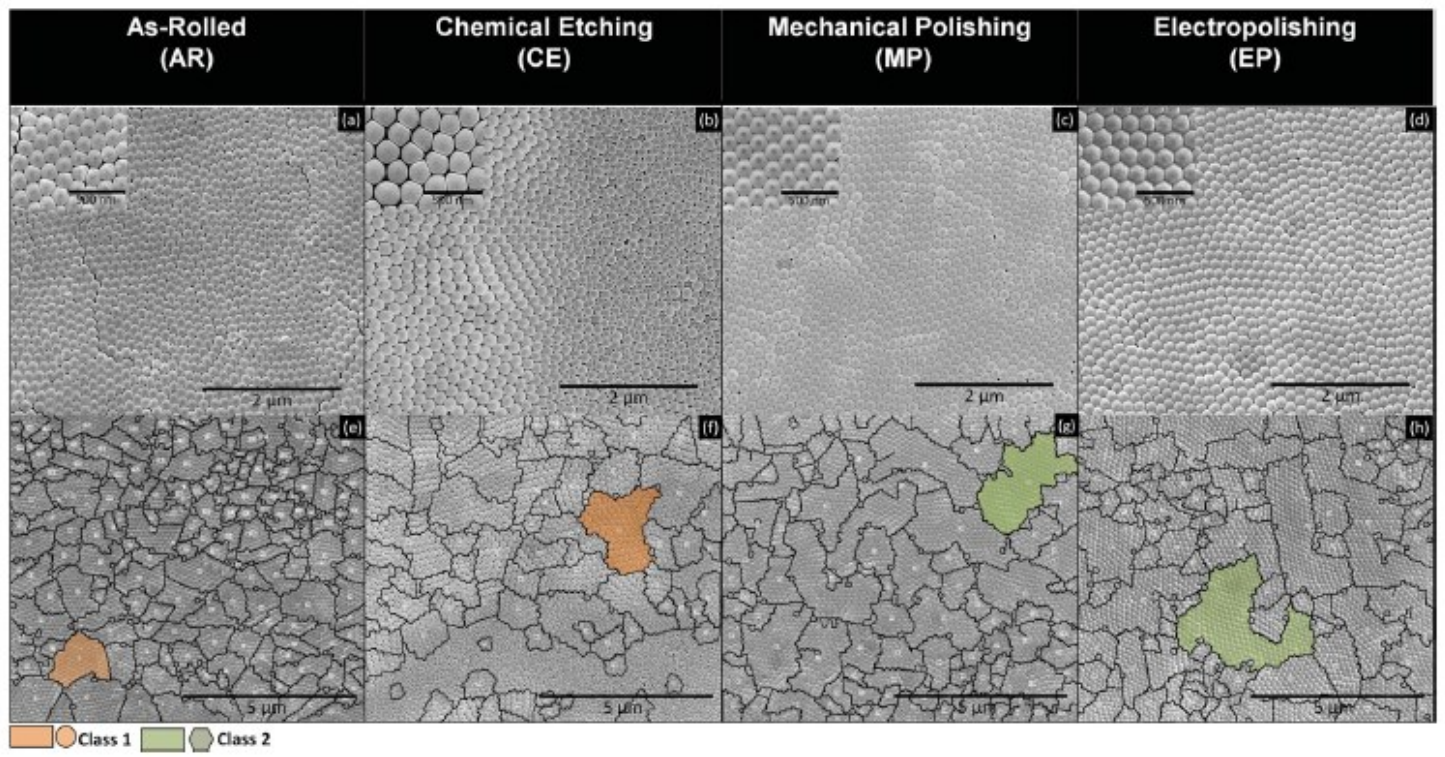

Fig. 13 SEM images of the TiO2 NT bottoms from the different samples: (a) AR, (b) CE, (c) MP and (d) EP. For each sample, a SEM image was analyzed [(e), (f), (g) and (h)]: the domain areas $\left(\mu \mathrm{m}^{2}\right)$ were delimited, labeled (not readable small white labels within each domain) and the maximum domain area in the image colored with the corresponding class types (class 1 and class 2). 


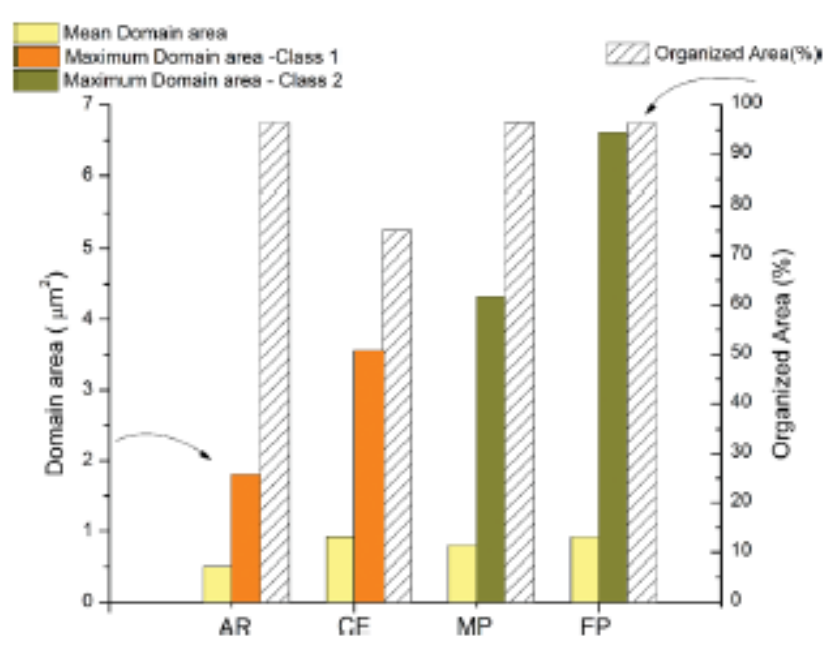

Fig. 14 Statistical results of the analyzed NT bottom SEM images for the different samples, indicating the results obtained for the mean and maximum domain areas (class 1 and class 2) and the organized area (\%).

\section{NT density: number of NTs per unit area and NT diameters.}

Fig. 15 shows the results of the NT density and diameter (D) of the studied samples. The average diameter was estimated from the histogram of the size distribution, which was then fitted to a log-normal distribution (inset of Fig. 15). The decrease of Rq in the MP and EP samples leads to a decrease in the NT density since D increases. The NT density is therefore also determined by the $\mathrm{Ti} \mathrm{Rq}$ prior to the anodization. According to this relationship one would expect the EP sample, which has a slightly higher Rq than the MP sample, to have a higher NT density (and a slightly smaller D). The observed smaller density of the EP sample is related to the fact that this sample has NT arrays with less space between the hexagon corners than the MP sample. It should be noted that the EP sample surface showed a pattern with small ripple-like structures, which act as nucleation points on the valleys, forcing the nucleation to occur simultaneously in an organized manner. Since this periodical structure has a spacial period of $\lambda=97 \mathrm{~nm}(D \approx$ $157 \mathrm{~nm}$ ), each valley behaves as a single nucleation site. The NTs then turn to be more packed and with slightly higher diameters due to an increase of the current - owing through these sites. Thus, almost no competition exists between the NTs, resulting in optimized structures. As for the CE sample, the darker disorganized regions correspond to NTs with small diameters $(D=130 \mathrm{~nm}$ and $\sigma=29.4 \mathrm{~nm}$ ) and a NT density of 57 NTs per $\mu \mathrm{m} 2$. On the other hand, the highly organized areas (zone 2) have NTs with much larger D (196 nm and $\sigma=0.286 \mathrm{~nm}$ ) and a density of 32 NTs per $\mu \mathrm{m} 2$. This increase of the diameter is associated with the surface topography that increases the electric field in the valley-sites.

In the case of the CE sample, the previously mentioned trend of increasing D (and consequently the NT density) with decreasing Rq (Fig. 15) is not completely valid. Although non-organized areas indeed show the expected trend, in the organized areas, the Rq increase leads to an increase in D. Remember the special behavior of this sample (in subsection "The particular case of high roughness."), where two different areas cause different anodization conditions and consequently NT organization, diameters and 
density. Indeed, Raja et al. suggested that pore ordering originates from the effect of local surface perturbations that causes the increase of the strain energy, i.e. electrostriction, electrostatic and recrystallization stresses at the valleys. ${ }^{51}$ Since the surface energy acts as a stabilizing force on a plane surface, when perturbed by a sinusoidal morphology the energy density varies across the hills and valleys, showing higher stress concentration and strain energy on the latter and leading to $\mathrm{F}^{-}$migration and adsorption where the NT structure first occurs. Thus, due to the periodic perturbations of such ripples, the electric field is higher on the valleys, which results in NT growth in these sites. Therefore, one of the main achievements of this study is the experimental proof that the NT diameter is not only associated with the anodization potential but also with the Ti surface conditions.

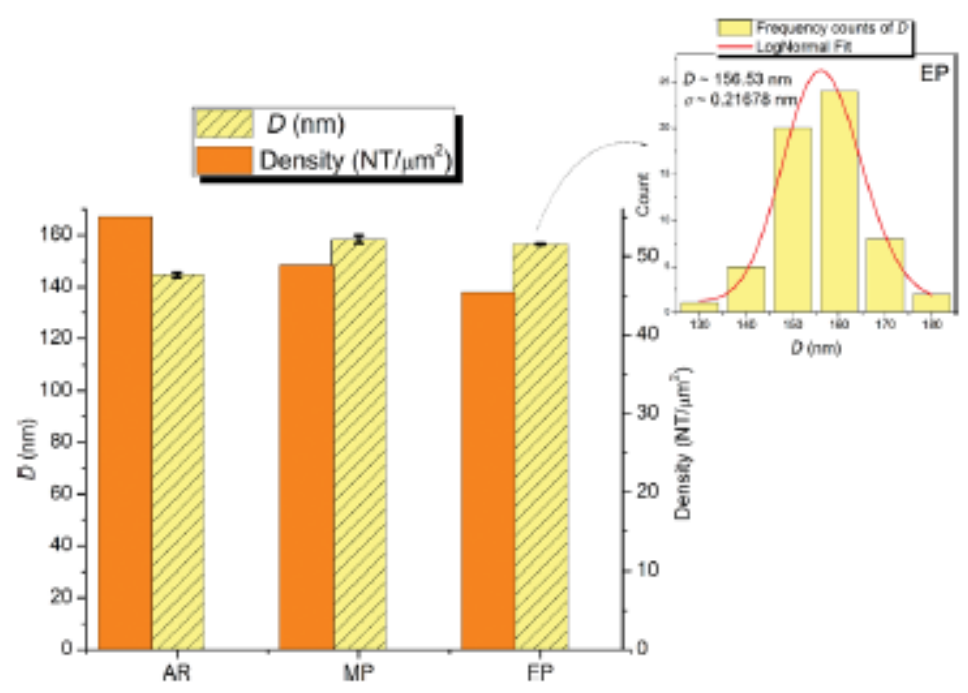

Fig. 15 Density number of NTs per area (NTs per $\mu \mathrm{m}^{2}$ ), NT diameter (D) and standard deviation $(\sigma)$ for the AR, MP and EP samples. $D$ and $\sigma$ were calculated by performing a histogram of the NT diameter distribution and a log-normal fit; the upper inset shows the NT D histogram and log-normal fit of the EP sample.

\section{Electropolishing as a one step anodization route.}

MP and EP pre-treatments led to the best results, both regarding the total NT length and their level of organization. Nevertheless, to achieve MP samples with a high level of smoothness $(\mathrm{Rq} \approx 1 \mathrm{~nm})$ is a long and expensive process with low reproducibility. In this context, the EP process becomes more attractive and easy to perform. Despite not leading to the smallest $\mathrm{Rq}$ values, the EP pre-treatment actually facilitates NT growth and organization due to the intrinsic formation of the periodic ripples pre-patterned in the Ti surface. This process is similar to the two-step anodization where the dimple pattern left by the first anodization is crucial to achieve the ideal hexagonal ordering in the second step. ${ }^{34}$ These dimples on the Ti surface act as initiation sites for tube growth and thus may well be a pre-condition to obtain extended order. 
Macak et al. showed that performing two step anodization on Ti foils significantly improves the hexagonal ordering, decreasing the number of packing errors (pentagons, heptagons, etc.), although it further increases the number of anodization steps. ${ }^{26}$ In the present work, we explained that MP and EP pre-treatments successfully led to the best results: NT length and their level of organization, avoiding the second anodization. However, to achieve MP samples with a high level of smoothness $(\mathrm{Rq} \approx 1 \mathrm{~nm}$ ) is a long, hard and expensive process with low reproducibility. The use of EP pre-treatment leads to highly hexagonal self-ordered samples with large domains of organization at a reduced time and cost. In this context, the EP process becomes more attractive and easy to perform.

Furthermore, the degree of order can be evaluated calculating the porosity (P) of the NT arrays. For an ideal hexagonal arrangement of pores, $\mathrm{P}$ can be estimated using:

$$
P=\frac{2 \pi}{\sqrt{3}}\left(\frac{D_{\mathrm{p}} / 2}{D_{\mathrm{int}}}\right)^{2},
$$

where the pore diameter is $D p=2 r$ and Dint is the interpore distance. Using ImageJ we calculated a mean wall thickness of $w \approx 51.78 \mathrm{~nm}$ (SEM image of the MP sample of Fig. 5) and with the external diameter previously extracted from the histogram analyses $(D=158.3 \mathrm{~nm}$ ), we obtain the internal diameter $\mathrm{Dp}=54.78 \mathrm{~nm}$. Due to the hexagonal geometry, we considered the interpore distance (Dint) equal to the external diameter ( $D=$ Dint). Using eqn (3), $D p=54.8 \mathrm{~nm}$ and Dint $=158.3 \mathrm{~nm}$, we obtain a porosity of $\mathrm{P} \approx$ $10.8 \%$. The NT templates thus present a porosity close to the $10 \%$ benchmark reported for the self-organized-regime in AAO templates. ${ }^{32}$

The closely packed hexagonal structure with a degree of order comparable to that of AAO templates was not reported so far for $\mathrm{TiO} 2$ templates. Comparing the results achieved in this work with the already reported domains of $1.83 \mu^{2}$ with $20 \%$ of porosity, ${ }^{25}$ the proposed approach contributes to the improvement of both ordered (HCP) domain areas and porosity of hexagonal arrays of TiO2 nanotubes.

\section{Conclusions}

By bonding the formation and growth mechanisms of the TiO2 NTs with the analyses of the anodization curves, this work reveals a new overriding anodization parameter, the Ti surface roughness, capable of inducing a higher growth rate (extended NT length) and improved self-organization.

The analyses of the anodization curves, $j(t), d j / d t$ and $Q(t)$, clearly revealed the importance of the surface topography details: ripple-like (EP) and random broad (AR) valleys promote effective nucleation spots and thus the earlier emergence of NT nucleation and lower $\Delta$ th than a smoother topography (MP). Furthermore, the samples with lower $\mathrm{Rq}$ (and the presence of small nucleation sites) show a uniform enhancement 
of the growth rate as well as a highly organized array. With a lower surface Rq, we obtain a more homogeneous electric field over the sample surface, causing a thinner $\delta b$ that promotes ionic electromigration $\left(\mathrm{F}^{-}\right.$and $\left.\mathrm{O}^{2-}\right)$ through $\delta \mathrm{b}$. This is fundamental for the dissolution/oxidation balance in steady state anodization. Indeed, samples with smoother surface $\mathrm{Rq}(\mathrm{MP}$ and $\mathrm{EP}$ ) presented higher $\mathrm{j}(\mathrm{t})$ and $\mathrm{Q}(\mathrm{t})$, a thinner $\delta \mathrm{b}$ and $\mathrm{a}$ smaller $\delta b(t)$ increase during the anodization. Furthermore, a smoother surface promotes a higher mobility of $\mathrm{F}^{-}$ions in the dominant anodization processes, promoting greater accessibility of $\mathrm{F}^{-}$ions to reach the Ti surface (and thus favoring the dissolution processes), overcoming the main cause of increasing $\delta \mathrm{b}$. Also, the additional chemical effects (namely pH decrease throughout the anodization), which limit the NT growth and lead to a non-steady state anodization, are delayed since the anodization growth limit is postponed. We observed that the degree of ordering is highly extended by decreasing Rq. We developed a new method to quantify the degree of such organization including the measurement of HCPA domain areas. Large areas with an ideal structure represented by a HCPA of hexagonally arranged cells were obtained ( 4.32 and $6.61 \mu \mathrm{m}^{2}$ for MP and EP samples respectively), two times larger than those previously reported. Furthermore, the obtained TiO2 NT array structures present a degree of order comparable to that of AAO templates (a porosity of $11 \%$ ).

Finally, we emphasize that by using a single anodization step with the presented optimized pre-treatments one can produce high quality TiO2 NT arrays, with large organized domains at a reduced time and cost, making it a straightforward application on Ti foils.

\section{Acknowledgements}

A. Apolinário acknowledges the financial support under the Portuguese Foundation for Science and Technology (FCT) project PTDC/EQU-EQU/107990/2008. L. Andrade, D. C. Leitão and C. T. Sousa are also grateful to FCT for their Post-Doc fellows (references: SFRH/BPD/74944/2010, SFRH/BPD/72359/ 2010 and SFRH/BPD/82010/2011, respectively). J. Ventura acknowledges financial support through FSE/POPH. The authors acknowledge funding from FCT through the PTDC/EQU-EQU/101397/2008 project and the Associated Laboratory - IN. The authors also acknowledge Operation NORTE-070124-FEDER-000070Multifunctional Nanomaterials funded by FEDER and CCDRN.

\section{Notes and references}

1. R. Krol,Y.Liang and J.Schoonman,J. Mater. Chem.,2008, 18, 2311; P. Song, X. Zhang, M. Sun, X. Cui and Y. Linb, Nanoscale, 2012, 4, 1800.

2. X. Lu, G. Wang, T.Zhai, M. Yu, J. Gan, Y.Tong and Y. Li, Nano Lett., 2012, 12, 1690.

3. P.Xiao, B. B.Garcia, Q.Guo, D.LiuandG.Cao, Electrochem. Commun., 2007, 9, 2441.

4. B. O'Regan and M. Gratzel, Nature, 1991, 353, 737; M. Gratzel, Nature, 2001, 414, 338. 
5. P. Roy, D. Kim, K. Lee, E. Spieckerb and P. Schmuki, Nanoscale, 2010, 2, 45.

6. V. Zwilling, M. Aucouturier and E. Darque-Ceretti, Electrochim. Acta, 1999, 45(6), 921.

7. M. Paulose, H. E. Prakasam, O. K. Varghese, L. Peng, K. C. Popat, G. K. Mor, T. A. Desai and C. A. Grimes, J. Phys. Chem. C, 2007, 111, 14992; M. Paulose, K. Shankar, Yoriya, H. E. Prakasam, O. K. Varghese, G. K. Mor, A. Latempa, A. Fitzgerald and C. A. Grimes, J. Phys. Chem. B, 2006, 110,16179.

8. K. Shankar, G. K. Mor, H. E. Prakasam, S. Yoriya, M. Paulose, O. K. Varghese and C. A. Grimes, Nanotechnol., 2007, 18, 065707.

9. V.Vega, M. A. Cerdeira, V. M. pride, D. Alberts, N. Bordel, R. Pereiro, F. F. Mera, S. Garcia, M. Hernandez-Velez and M. Vasquez, J. Non-Cryst. Solids, 2008, 354, 5233.

10. E. Balaur, J. M. Macak, L. Taveira and P. Schmuki, Electrochem. Commun., 2005, 7, 1066.

11. H. Yin, H. Lui and W. Z. Shen, Nanotechnol., 2010, 21, 035601.

12. S. Sreekantan, K. A. Saharudin, Z. Lockman and T. W. Tzu, Nanotechnol., 2010, 21, 365603.

13. S. So, K. Lee and P. Schmuki, J. Am. Chem. Soc., 2012, 28, 11316; S. So, K. Lee and P. Schmuki, Chem. -Eur. J., 2013, 19, 2966.

14. S. Berger, R. Hahn, P. Roy and P. Schmuki, Phys. Status Solidi B, 2010, 247, 2424.

15. M. Macak, H. Tsuchiya and P. Schmuki, Angew. Chem., Int Ed, 2005, 44, 2100.

16. L.Sun,S.Zhang, X. W.SunandX. He, J.Electroanal. Chem., 2009,637,6.

17. D. Guan and Y. Wang, Nanoscale, 2012, 4, 2968.

18. X.TangandD. Li,J. Phys. Chem. C, 2009,113, 7107.

19. S. Berger, J. Kunze, P. Schmuki, D. LeClere, A. T. Valota, P. Skeldon and G. E. Thompson, Electrochim. Acta, 2009, 54, 5942.

20. Q. Y. Cai, M. Paulose, O. K. Varghese and C. A. Grimes, J. Mater. Res., 2005, 20, 230.

21. V. Vega, V. M. Prida, M. Hernández-Vélez, E. Manova, P. Aranda, E. Ruiz-Hitzky and M. Vázquez, Nanoscale Res. Lett., 2007, 2, 355.

22. V. M. Prida, V. Vega, P. Aranda, M. Vázquez and E. Ruiz- Hitzky, J. Magn. Magn. Mater., 2007, 316, 110.

23. S. Kim, B. G. Lee and J. Choi, Bull. Korean Chem.Soc., 2011, 32, 3730.

24. X. Yuan, M. Zheng, L. Ma and W. Shen, Nanotechnol., 2010, 21, 405302.

25. G. D. Sulka, J. Kapusta-Kołodziej, A. Brzozka and M. Jaskuła, Electrochim. Acta, 2010, 55, 4359.

26. J. M. Macak, S. P. Albu and P. Schmuki, Phys. Status Solidi RRL, 2007,1,181.

27. Y.Shin andS. Lee, Nano Lett., 2008, 8, 3171.

28. B. G. Lee, S. Y. Hong, J. E. Yoo and J. Choi, Appl. Surf. Sci., 2011, 257, 7190.

29. K. Lu,Z. Tian and J. A. Geldmeier., Electrochim. Acta, 2011, 56, 6014.

30. K.Zhu, T. B. Vinzant, N. R. NealeandA.J. Frank, Nano Lett., 2007, 7, 3739.

31. S.P. Albuand P. Schmuki, Phys. Status SolidiRRL, 2010, 4, 151.

32. K. Nielsch, J. Choi, K. Schwirn, R. B. Wehrspohn and Gosele, Nano Lett., 2002, 2, 677.

33. Y. Li, X. Yu and Q. Yang, J. Sens., 2009, 2009, 402174.

34. F. Y.Li, L. Zhangand R. M. Metzger, Chem. Mater., 1998,10, 2470.

35. H. Masuda and K. Fukuda, Science, 1995, 268, 1466. 
36. D. C. Leitao, A. Apolinario, C. T. Sousa, J. Ventura, J. B. Sousa, M. Vazquez and J. P. Araujo, J. Phys. Chem. C, 2011, 115, 8567.

37. K. F. Albertin, A. Tavares and I. Pereyra, Appl. Surf. Sci., 2013, 284, 772.

38. B. Chen, K. Lu and Z. Tian, J. Mater. Chem., 2011, 21(24), 8835.

39. H. Masuda, H. Masuda, H. Asoh, M. Watanabe, K. Nishio, M. NakaoandT. Tamamura, Adv. Mater., 2001,13,189.

40. S. Singh, W. R. T. Barden and P. Kruse, ACS Nano, 2008, 2, 2453.

41. Q. Chen, D. Xu, Z. Wu and Z. Liu, Nanotechnol., 2008, 19, 365708.

42. S. Bandyopadhyay, A. E. Miller, H. C. Chang, G. Banerjee, V. Yuzhakov, D. F. Yue, R. E. Ricker, S. Jones, J. A. Eastman, E. Baugher and M. Chandrasekhar, Nanotechnol., 1996, 7, 360-371.

43. V. V. Yuzhakov, H. C. Chang and A. E. Miller, Phys. Rev. B, 1997, 56, 12608.

44. V.V.Yuzhakov, P.V.Takhistov,A.E. MillerandH.C.Chang, Chaos, 1999, 9, 62.

45. L. B. Kong, Y. Huang, Y. Guo and H. L. Li, Mater. Lett., 2005, 59, 1656.

46. D. Landolt, P. F. Chauvy and O. Zinger, Electrochim. Acta, 2003, 48, 3185.

47. N. Cabrera and N. F. Mott, Rep. Prog. Phys., 1948-1949, 12,163.

48. V. P. Parkhutik and V. I. Shershulsky, J. Phys. D: Appl. Phys., 1992, 25, 1258.

49. W. S. Rasband, ImageJ, US National Institutes of Health, Bethesda, Maryland, USA, http://rsb.info.nih.gov/ij/, 1997-2009.

50. W. Ruff, Metall. Mater. Trans. B,1974,5,601.

51. K. S. Raja, M. Misra and K. Paramguru, Electrochim. Acta, 2005, 51, 154. 